\title{
SOLPS-ITER modeling of beryllium trace impurity in ITER
}

\author{
Sergei Makarov ${ }^{1, *}$, Elizaveta Kaveeva ${ }^{1}$ \\ ${ }^{1}$ Peter the Great St.Petersburg Polytechnic University (SPbPU), Polytechnicheskaya 29, 195251, \\ St.Petersburg, Russia
}

\begin{abstract}
Beryllium transport modeling was carried out in the trace impurity regime by SOLPS-ITER code for tokamak ITER. It is valuable for developing of diagnostics protection from beryllium deposition. TRIM reflection model and Bogdanskii formula sputtering model were applied. The idea is to show possibility of using this code for beryllium transport. This code has key advantage comparing to other codes, which study beryllium transport, because in it the main plasma and impurities are described self-consistently. Therefore, if beryllium affect the background plasma, only this code can take this effect into account. Test calculations were performed with suppressed sputtering yield by 10 times, to reach trace impurity regime. Main features of behavior of beryllium in the tokamak were studied, however under restriction of static wall conditions. Sources, sinks, fluxes and density distribution were determined. In this test calculation plasma state kept unchanged as expected. Distribution of impinging and absorbed fluxes on the outer divertor plate were established in the position of divertor diagnostics. Main ways for the further improvement are suggested.
\end{abstract}

\section{Introduction}

Finding new sources of energy is one of the most challenging problems these days. Fusion is a relatively reliable way to solve it. ITER is the most advanced project in this area. ITER is a tokamak, and it will be built with beryllium first wall and tungsten divertor [1]. Beryllium will be sputtered and redistributed in the vacuum chamber. Beryllium will also deposit on the mirrors of ITER diagnostics. It is important to keep them clean during whole discharge to get correct data from diagnostics. One of the approaches to avoid this influence is so called cleaning discharge [2]. However, a beryllium flux, which cleaning discharge system can handle with, is strictly limited. There is no possibility to check this technology entirely on another setup, because of unique ITER parameters. Accordingly, the simulations of beryllium transport by SOLPS-ITER code in the ITER chamber were started in order to estimate beryllium flux on diagnostics mirrors.

The test calculation was made to confirm applicability of this code for beryllium transport. Earlier the SOLPS-ITER code was used for modeling of different tokamaks with

* Corresponding author: sergey.o.makarov@gmail.com 
different sorts of impurities (such as He, N, Ne, C) such as ITER [3], Globus-M [4], ASDEX Upgrade [5], JET [6], DIII-D [7], Alcator C-Mod [8] and others.

There are several codes, which describe beryllium transport, like WallDYN [9] and ERO [10, 11]. However, these codes work with static background plasma. Therefore, there is no any feedback on the plasma parameters by beryllium density and flows. Nevertheless, significant amount of beryllium impurity in the tokamak can change background plasma. SOLPS-ITER calculates background plasma and impurities self-consistently. Moreover, these codes use as a background the results of SOLPS-ITER modeling without drifts and electrostatic potential description. At the same time, the drift flows proved important for redistribution of impurities in ASDEXUpgrade [12].

The SOLPS-ITER code is developed by the ITER Organization $[13,14]$ on the base of codes SOLPS4.3 [15] and SOLPS5.2 [16] and now used as a main instrument for edge plasma fluid modeling by ITER Organization. This code includes description of drifts and currents, necessary for self-consistent description of plasma electrostatic potential. The new modeling described further is fulfilled with drifts and currents turned on.

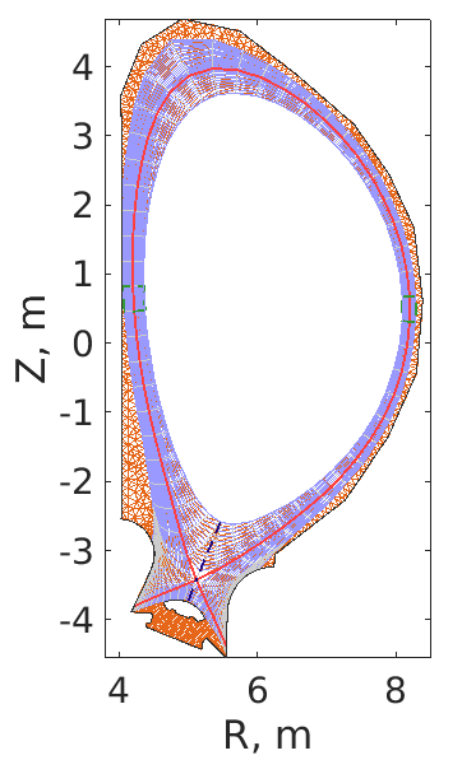

Fig. 1. Calculation grid in the ITER tokamak simulation. Purple indicates the grid of code B2. Orange indicates the grid of the EIRENE code. Red is a separatrix.

\section{Method}

\subsection{Model description}

The SOLPS-ITER code is structurally divided into two parts. The first part, the hydrodynamic code B2, is responsible for calculating transport of ions, parallel momentum, and energy in the computational grid B2. The second part, the Monte Carlo EIRENE code, is responsible for calculating of distribution of the neutral atoms and molecules in the EIRENE computational grid, which is built into the B2 computation area, and is continued to the material surface (Figure 1). Moreover, the EIRENE code is responsible for the interaction of ions (based on data from B2), atoms and molecules with the material surface [17].

The interaction with the surface is divided into two separate tasks: reflection modeling and sputtering modeling. Reflection modeling is further subdivided into two more mechanisms: a) calculation of reflection using the TRIM database for the reflection coefficients $[17,18,19]$ and b) absorption of particles with further release of that particles with the energy equal to wall temperature (monoenergetic particles), and cosine angular distribution for particles, which were not reflected. For beryllium, only the "a" mechanism was included. Thus, if beryllium was not reflected by the TRIM mechanism (about 10\%), it was dropped out of consideration. Reflection for ions (recycling), unlike neutral particles, occur on the B2 boundaries.

Sputtering from beryllium surfaces is the only source of beryllium in this calculation (there is no sputtering from the tungsten surfaces where beryllium deposited). Sputtering is calculated using the Bogdanskii formula $[17,20,21]$. For the test calculation, the sputtering 
coefficient was multiplied by 0.1 , since in this case the amount of beryllium in the plasma was insignificant and its presence did not affect the discharge parameters. Thus, the calculation occurred in the trace impurity regime.

\subsection{Discharge parameters}

The composition of the plasma was: D, He, $\mathrm{Be}, \mathrm{Ne}$. Deuterium and neon was puffed into the plasma from the puffing system in ITER $\left(\mathrm{F}_{\mathrm{D} 2}=1.95 \times 10^{23} \mathrm{~s}^{-1} ; \mathrm{F}_{\mathrm{Ne}}=2 \times 10^{20} \mathrm{~s}^{-1}\right)$. Additionally, the flow of deuterium corresponding to NBI from the core region was imposed. Helium appeared in the core (in the real discharge by DT reaction) $\left(\mathrm{F}_{\mathrm{D}+}=\right.$ $\left.10^{22} \mathrm{~s}^{-1} ; \mathrm{F}_{\mathrm{He}+2}=10^{20} \mathrm{~s}^{-1}\right)$. Power came from the core region of the plasma and was equally divided between electrons and deuterium: $\mathrm{Pe}_{\mathrm{e}}$ $=50 \mathrm{MW} ; \mathrm{P}_{\mathrm{i}}=50 \mathrm{MW}$.

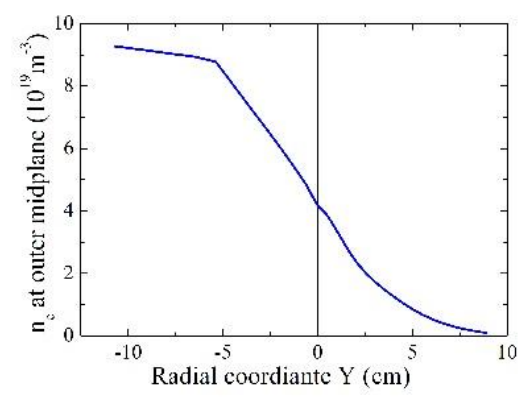

Fig. 3. Plasma density in the outer midplane.

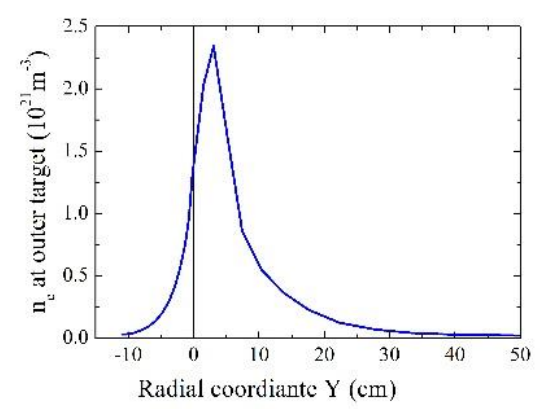

Fig. 5. Plasma density on the outer divertor plate.

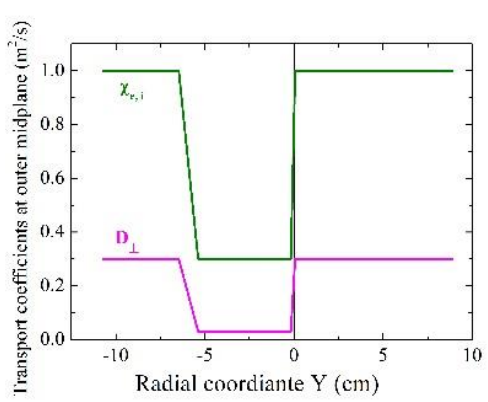

Fig. 2. Coefficients of anomalous transport in the outer midplane.

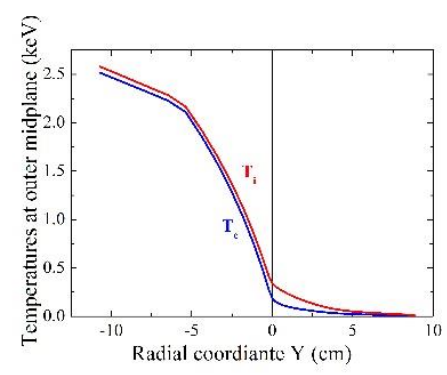

Fig. 4. Temperature of electrons and ions in the outer midplane.

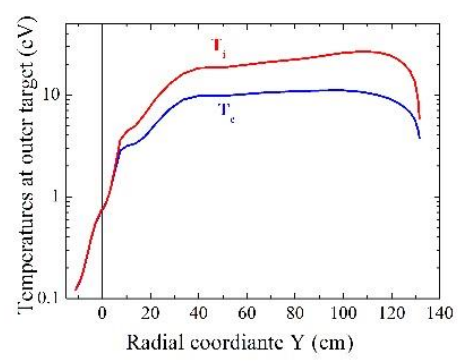

Fig. 6. The temperature of electrons and ions on the outer divertor plate.

Pumping for all types of particles on the pumping surface (under Dome) with a probability of $0.72 \%$ for impinging particle was imposed. Coefficients of anomalous transport (be given as input parameter) corresponded to H-mode (Figure 2). The neutral pressure under the dome was $10 \mathrm{~Pa}$, the neon concentration at the separatrix was $2 \%$. Main discharge parameters are shown on figures 3-6. 


\section{Results and Discussion}

Global Be balance are represented by following fluxes: $F_{\text {Be sputtered atoms surf }}=2.46 \times 10^{19} \mathrm{~s}^{-1}$ (sputtered by atoms and molecules); $\mathrm{F}_{\text {Be sputtered atoms B2 }}=1.07 \times 10^{19} \mathrm{~s}^{-1}$ (sputtered by ions); $\mathrm{F}_{\mathrm{Be}}$ absorbed atoms surf $=3.55 \times 10^{18} \mathrm{~s}^{-1} ; \mathrm{F}_{\mathrm{Be}}$ absorbed atoms $\mathrm{B} 2=1.28 \times 10^{18} \mathrm{~s}^{-1} ; \mathrm{F}_{\mathrm{Be}}$ absorbed ions $\mathrm{B} 2=2.93 \times 10^{19} \mathrm{~s}^{-}$ ${ }^{1}$. The main source of beryllium is situated on a material wall, and the main sink is deposition

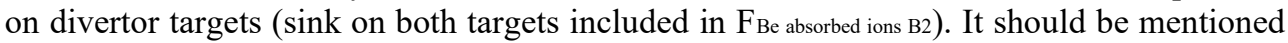
that the instantaneous values of the sputtered flows compensate values of the absorbed flows only approximately because of time variations. However, flows averaged for a sufficiently long period of time must compensate each other strictly. Figure 7 shows density distribution of beryllium ions calculated using B2 code. Figure 8 shows density distribution of beryllium atoms computed using EIRENE code. Huge amount of beryllium accumulates near divertor targets. Beryllium sputtered on the first wall is dragged to the divertor region by main ions fluxes, which form in the SOL.

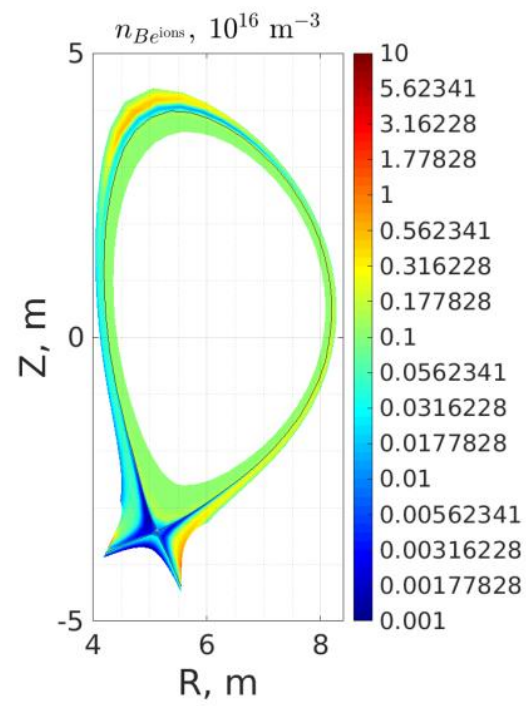

Fig. 7. Two-dimensional distribution of beryllium ions.

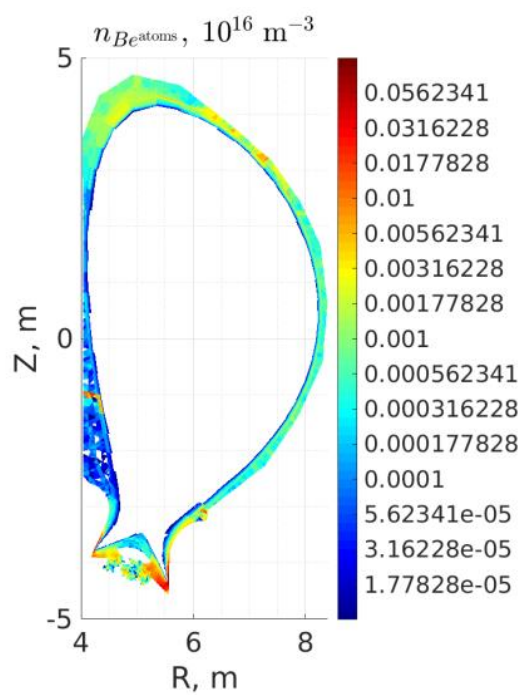

density Fig. 8. Two-dimensional density distribution of beryllium atoms.

Figures 9 and 10 show the flux densities of ions and beryllium atoms, respectively, in the region of the outer divertor. Beryllium is reflected from divertor plate, ionizes near the plate and forms additional ion flux on the target. As a result, near the divertor target ion flow increase dramatically (figure 9). Accordingly, high density of neutral Be was observed near the targets and in the private region (under X-point) (figure 8), where beryllium was transferred by neutral fluxes reflected from the plate (figure 10).

Figures 11 and 12 show the main flows of beryllium on the first wall and on the outer divertor target, respectively. The maximum of sputtering occurres near the surface number 20 , where D2 puffing was set and sputtering in this area is most effective. On the outer divertor plate about $90 \%$ of beryllium is reflected, however beryllium flux is so significant that sink (other 10\%) compensate first wall sputtering. 


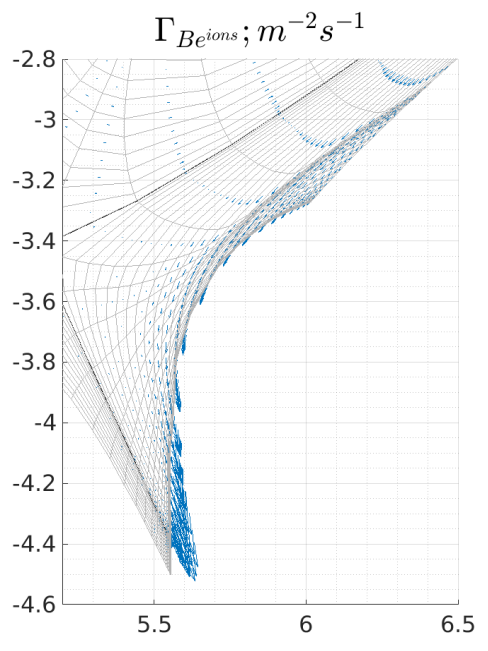

Fig. 9. Flux density of beryllium ions.

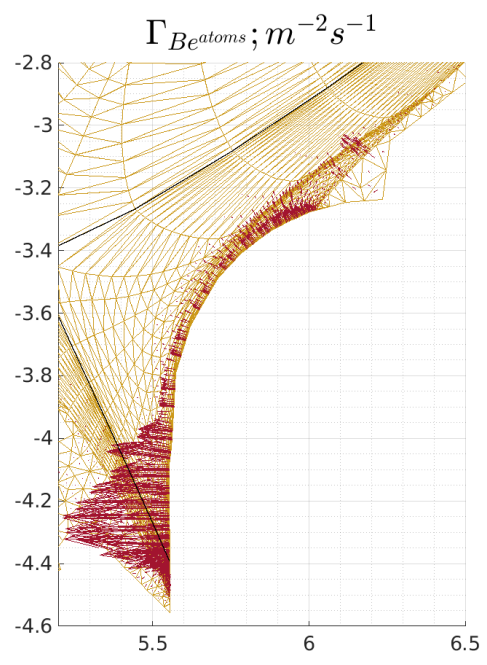

Fig. 10. Flux density of beryllium atoms.

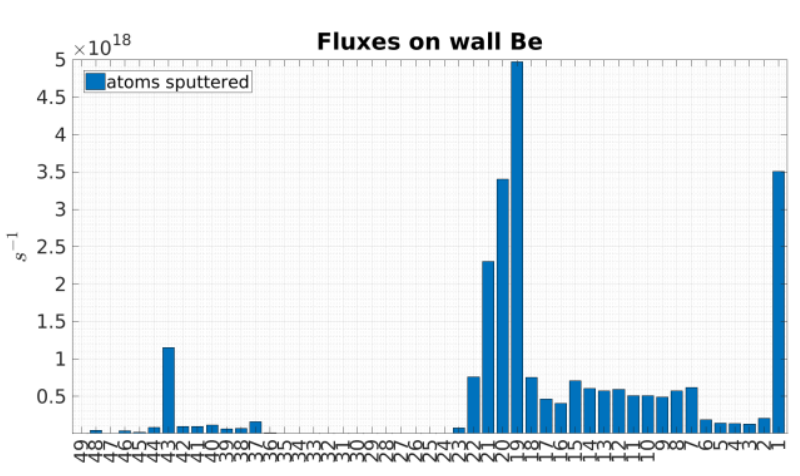

Fig. 11. Flows of beryllium atoms from the surfaces on the first wall (the numbers of material surfaces are plotted along the horizontal axis, see Figure 13).

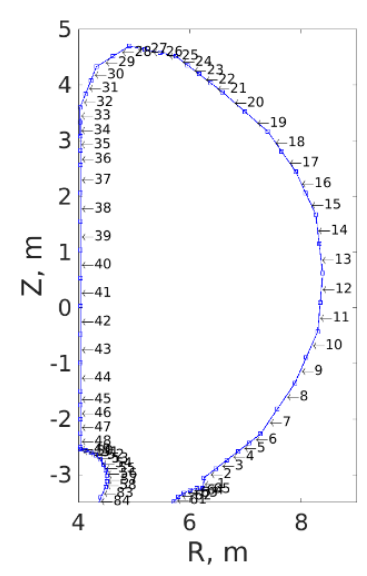

Fig. 12. Numbers of material surfaces (not the same numbers on the figure 15)

\section{Conclusion}

This calculation showed applicability of SOLPS-ITER for modeling beryllium transport. Trace impurity regime for beryllium was modeled and parameters of so called background plasma did not change. In this regime beryllium fluxes were studied. The steady-state conditions were reached, however only in assumption that redeposition area did not become a new source of beryllium, and it only was a sink. Distribution of sputtered flux on the first wall and incident beryllium flux on the outer divertor target were obtained. In the further study $100 \%$ sputtering should be included. Furthermore, re-erosion should be considered in the improved model. Accordingly, accumulation beryllium on the tungsten target have to be 
included in the SOLPS-ITER code. Dependence on the temperature and 3D geometry of first wall and divertor plate will also be valuable amendment to present surface interaction models. Therefore, these problems will be considered in the future. All these challenges can change beryllium behavior in the tokamak.

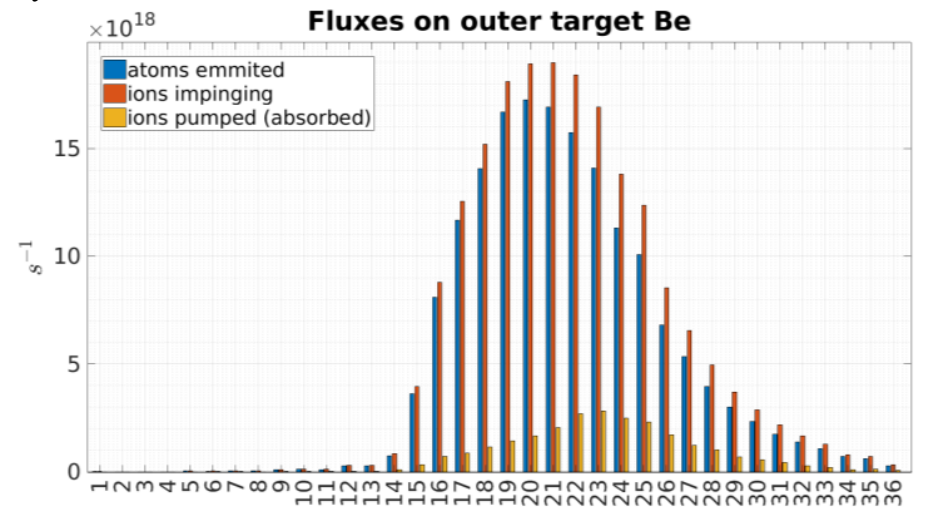

Fig. 13. Flows of atoms and ions of beryllium on the outer divertor plate (the number of surfaces (grid-surface boundaries) are plotted along the horizontal axis, see Figure 15).

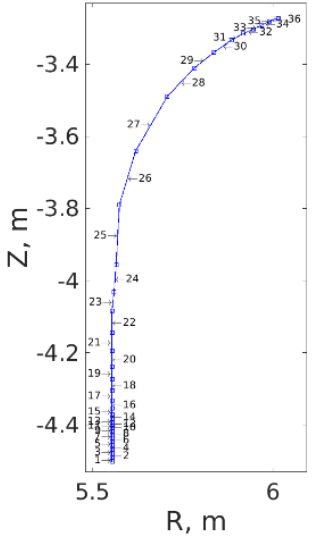

Fig. 14. Number of surfaces (grid-surface boundaries) on the outer divertor plate. Separatrix between 12 and 13 cells.

As an additional study, it will be interesting in the future to compare patterns of beryllium transport calculated by SOLPS-ITER code and by WallDYN and ERO, since these codes use considerably different transport models: fluid modeling for SOLPS-ITER and Monte-Carlo modeling of impurity ion for WallDYN and ERO.

This work was carried out at Peter the Great St. Petersburg Polytechnic University under the support of the Russian Science Foundation, grant no. 17-12-01020. Numerical calculations were performed at the Polytechnic supercomputer center at Peter the Great St. Petersburg Polytechnic University. The views and opinions expressed herein do not necessarily reflect those of the ITER Organization. Also, the work was carried out under contract no. 17706413348180000850/143602802 with Ioffe Physical-Technical Institute of the Russian Academy of Sciences.

\section{References}

1. R.A. Pitts et al J. Nucl. Mater. 415 S957-S964 (2011)

2. E.E. Mukhin et al Nucl. Fusion 52013017 (2012)

3. V. Rozhansky, E.Kaveeva, I. Veselova, S. Voskoboynikov, and D. Coster Contrib. Plasma Phys. 56 6-8 587 - 591 (2016)

4. E. Vekshina et al Plasma Phys. Control. Fusion 58085007 (2016)

5. I. Senichenkov, E. Kaveevaa, V. Rozhanskya, E. Sytovaa, I. Veselovaa, S. Voskoboynikova, and D. Coster Technical Physics Letters 44 No. 3, pp. 255-259 (2018)

6. L Aho-Mantila et al Plasma Phys. Control. Fusion 59035003 (2017)

7. E.T. Meier et al Nuclear Materials and Energy 12 973-977 (2017)

8. W. Dekeyser et al Nuclear Materials and Energy 12 899-907 (2017)

9. K. Schmid et al Nucl. Fusion 55053015 (2015) 
10. D Borodin et al Phys. Scr. 2011014008 (2011)

11. D. Borodin et al Nuclear Materials and Energy 9 604-609 (2016)

12. V Rozhansky, E Kaveeva, I Senichenkov, E Sytova, I Veselova, S Voskoboynikov, D Coster. Electric fields and currents in the detached regime of a tokamak // Contributions to Plasma Physics - 58 540-546 (2018)

13. Bonnin X, Dekeyser W, Pitts R, Coster D, Voskoboynikov S and Wiesen S Plasma Fusion Res. 111403102 (2016)

14. Wiesen S et al J. Nucl. Mater. 463480 (2015)

15. Kukushkin A S et al Fusion Engineering and Design 86 2865-2873 (2011)

16. Rozhansky V, Kaveeva E, Molchanov P, Veselova I, Voskoboynikov S, Coster D, Counsell G, Kirk A, Lisgo S, ASDEX-Upgrade Team and MAST Team Nucl. Fusion 49 025007 (2009)

17. D. Reiter. The EIRENE Code User Manual Institut für Energie- und Klimaforschung Plasmaphysik Forschungszentrum Jülich (Dec 20, 2017)

18. W. Eckstein and D.B. Heifetz. Data sets for hydrogen reflection and their use in neutral transport calculations. MPI-Garching Report IPP 9/59, MPI-Garching, August 1986. J.Nucl.Mater. 145-147, p332 (1987)

19. G. Bateman. Distribution of neutrals scattered off a wall. PPPL Appl. Phys. Rep. No. 1, PPPL, (1980)

20. W. Eckstein, Garcia-Rosales C., J. Roth, et al. Sputtering data. MPI-Garching Report IPP-9/82, MPI-Garching, February (1993)

21. J. Roth and C. Gracia-Rosales. Nucl.Fus. 36, 12:1647, (1996) 